\title{
Sensitivities of future solar neutrino observatories to nonstandard neutrino interactions
}

\author{
Pouya Bakhtiø and Meshkat Rajaee \\ Institute for research in fundamental sciences (IPM), PO Box 19395-5531, Tehran, Iran
}

(Received 6 April 2020; accepted 3 August 2020; published 25 August 2020)

\begin{abstract}
We study the matter effect caused by nonstandard neutrino interactions (NSI) in the future solar neutrino experiments, DUNE, HK, and MICA. The upcoming reactor experiment, JUNO is expected to provide the most precise measurements of solar neutrino oscillation parameters and is going to open up the era of subpercent precision in the leptonic mixing sector of the Standard Model (SM). Assuming JUNO can measure $\Delta m_{21}^{2}$ and $\Theta_{12}$ by subpercent precision and assuming SM as the null hypothesis, we study the possibility to constrain NSI parameters by the future solar neutrino experiments such as DUNE, HK and MICA and present excluded region in NSI parameter space, $\epsilon_{N}$ and $\epsilon_{D}$. For this purpose, we study the effect of NSI on solar neutrino propagation in the Sun and Earth and explore the dependence of the day-night asymmetry on the NSI parameters. we also study the effect of NSI at the detector on the simulated data for these experiments.
\end{abstract}

DOI: 10.1103/PhysRevD.102.035024

\section{INTRODUCTION}

Neutrino oscillation is well established by the data from a plethora of neutrino experiments using solar, atmospheric, reactor, and accelerator neutrino experiments over the last two decades [1]. The standard three flavor neutrino oscillation paradigm (standard paradigm) includes three active neutrinos that are linear combinations of three neutrino mass states. In the standard paradigm, there are three mixing angles $\theta_{12}, \theta_{13}, \theta_{23}$, two mass-squared differences $\Delta m_{31}^{2}, \Delta m_{21}^{2}$ and one Dirac type $C P$ phase $\delta_{C P}$. Most of the oscillation parameters have been measured with fairly good precision [2-4]; However, there are some unknown quantities, namely, the value of the Dirac $C P$ phase $\delta_{C P}$, sign of $\Delta m_{31}^{2}>0$ and the octant of $\theta_{23}$. To determine the unknown neutrino oscillation parameters, experiments with high statistics, such as JUNO [5], T2HK [6], and DUNE [7], have been proposed.

One way to test the standard paradigm is to measure the neutrino oscillation probabilities and compare the observed pattern with the expectations from standard paradigm. If the expectations from standard paradigm do not match the observed patterns, one can deduce that there is some unknown new physics beyond standard paradigm.

Published by the American Physical Society under the terms of the Creative Commons Attribution 4.0 International license. Further distribution of this work must maintain attribution to the author(s) and the published article's title, journal citation, and DOI. Funded by SCOAP ${ }^{3}$.
The framework of nonstandard neutrino interaction (NSI) provides one model-independent way to extend the standard model to quantify new physics in the neutrino sector as well as explaining neutrino mass. NSI was explored as a solution to the solar neutrino problem [8], and their impact on the oscillations of solar neutrinos [9], atmospheric neutrinos [10], and accelerator neutrinos [11] have been explored in the literature. Moreover, several consequences of NSI to DUNE were also explored in $[12,13]$. Moreover, there is a tension between the masssquared difference obtained from the solar neutrino observations and the one from the KamLAND experiment. As studied in [14], one proposed solution is the sterile neutrino oscillation with the mass-squared difference of order of $\mathrm{O}\left(10^{-5}\right) \mathrm{eV}^{2}$, which is the so-called super light sterile neutrino scenario (SSNS). Another possibility is that the tension can be resolved by introducing the flavordependent NSI in neutrino propagation $[15,16]$.

The Jiangmen Underground Neutrino Observatory (JUNO), the future reactor experiment with the baseline of $50 \mathrm{~km}$ is going to provide the possibility to measure $\Delta m_{21}^{2}$ and $\theta_{12}$ to the percent level [17] while not sensitive to the NSI parameters due to its short baseline and low neutrino energy [18]. The potential of JUNO to test SSNS is studied in [19].

In this work, we investigate how well precision measurements of oscillation probabilities at JUNO can be used to probe the existence of NSI. We consider future solar neutrino experiments, DUNE and HK in addition to the proposed solar neutrino experiment, MICA and explore the potential of these experiments in resolving standard 
parameter degeneracies in the presence of NSI. Here we discuss the matter effect caused by NSI in the future solar neutrino experiments, using precision measurements of oscillation probabilities at JUNO.

Since solar neutrino oscillation probabilities are highly dependent on the NSI parameters due to the matter effect, with precise measurement of $\Delta m_{21}^{2}$ and $\theta_{12}$ by JUNO, we can investigate how well the future solar neutrino observatories can constrain nonstandard neutrino interaction parameters. Also, considering the day-night asymmetry of solar neutrino, we study the dependence of the day-night asymmetry on the NSI parameters. In addition, we explore the effect of NSI on the neutrino detection for HK and MICA detectors experiments which are water Cerenkov detectors [20].

For simplicity, we assume the same NSI couplings to electron, up quark, and down quark. As it is discussed in detail, assuming nonstandard couplings to electrons will affect the electron-neutrino scattering cross section and can lead to NSI at the HK and MICA detectors.

The paper is organized as follows. In Sec. II, we discuss the NSI Lagrangian and its effect on solar neutrino oscillation. In Sec. III, we discuss the details of different experiments and our simulation. In Sec. IV, we present our results. We briefly discuss our results in Sec. V.

\section{NEUTRINO OSCILLATION FRAMEWORK WITH NSI}

Neutral current (NC) NSI can be written as an effective four fermion operator

$$
\mathcal{L}_{\mathrm{NSI}}=-2 \sqrt{2} G_{F} \epsilon_{\alpha \beta}^{f P}\left(\bar{\nu}_{\alpha} \gamma^{\mu} \nu_{\beta}\right)\left(\bar{f} \gamma_{\mu} P f\right),
$$

where $f$ is a charged fermion, $P=(L, R)$ and $\epsilon_{\alpha \beta}^{f P}$ are dimensionless parameters encoding the deviation from standard interactions and $G_{F}$ is the Fermi coupling constant. Constraints on $\epsilon_{\alpha \beta}$ have been discussed in many references. For instance, there are bounds from atmospheric neutrinos [21-24], from $e^{+} e^{-}$colliders [25], from the compilation of various neutrino data [26,27], from solar neutrinos [28-30], from $\nu_{e} e$ or $\bar{\nu}_{e} e$ scatterings [30], from solar, reactor, and accelerator neutrinos [31,32]. In addition, NSI has been studied in the context of long-baseline experiments $[12,33,34]$.

NSI can be induced by the new physics beyond the standard model, by integrating out the heavier mediator fields which can generate the dimension-6 [35] and dimension-8 [36] effective operators. For a detailed review see Refs. [37,38].

The neutral current NSI (NC NSI) affect the neutrino oscillation in matter via forward elastic scattering. NC NSI also can affect the neutrino detection via neutrino electron scattering. In this work, we consider the effect of NSI on solar neutrinos for three cases: (i) through their propagation in the Sun, (ii) through their propagation in the earth, and (iii) by water Cherenkov detectors.

\section{A. Propagation of neutrinos in the Sun in the presence of NSI}

In the flavor basis, the flavor change of neutrinos through the propagation can be written as

$$
i \frac{d}{d x} \psi_{\nu}=H \psi_{\nu}
$$

where the total Hamiltonian includes the vacuum effect, standard matter effect or MSW effect and NSI matter effect

$$
H=H_{\mathrm{vac}}+H_{\mathrm{mat}}^{\mathrm{MSW}}+H_{\mathrm{mat}}^{\mathrm{NSI}}
$$

The vacuum term includes six parameters, $\Delta m_{21}^{2}, \Delta m_{31}^{2}$, $\theta_{12}, \theta_{13}, \theta_{23}, \delta_{C P}$ and is given by

$$
H_{\text {vac }}=U \operatorname{diag}\left(0, \frac{\Delta m_{21}^{2}}{2 E_{\nu}}, \frac{\Delta m_{31}^{2}}{2 E_{\nu}}\right) U^{\dagger}
$$

where $U$ is the standard Pontecorvo-Maki-NakagawaSakata mixing matrix, $U=R_{23} \Gamma_{\delta} R_{13} \Gamma_{\delta}^{\dagger} R_{12}$, where $R_{i j}$ represents a real rotation by an angle $\theta_{i j}$ in the $i j$ plane, $\Gamma_{\delta}=\operatorname{diag}\left(1,1, e^{i \delta}\right)$. The Hamiltonian of standard matter effect is given by $H_{\mathrm{mat}}^{\mathrm{MSW}}=\sqrt{2} G_{F} N_{e} \operatorname{diag}(1,0,0)$, where $N_{e}$ is the number density of electron in the medium. Moreover, the NSI matter effect is given by

$$
H_{\mathrm{mat}}^{\mathrm{NSI}}=\sqrt{2} G_{F} \sum_{f=e, u, d} N_{f}\left(\begin{array}{ccc}
\epsilon_{e e}^{f} & \epsilon_{e \mu}^{f} & \epsilon_{e \tau}^{f} \\
\epsilon_{e \mu}^{f *} & \epsilon_{\mu \mu}^{f} & \epsilon_{\mu \tau}^{f} \\
\epsilon_{e \tau}^{f *} & \epsilon_{\mu \tau}^{f *} & \epsilon_{\tau \tau}^{f}
\end{array}\right) .
$$

It is possible to define NSI parameter in the medium

$$
\epsilon_{\alpha \beta} \equiv \sum_{f=e, u, d}\left\langle\frac{N_{f}}{N_{e}}\right\rangle \epsilon_{\alpha \beta}^{f}=\epsilon_{\alpha \beta}^{e}+Y_{u} \epsilon_{\alpha \beta}^{u}+Y_{d} \epsilon_{\alpha \beta}^{d}
$$

where $Y_{\alpha}$ is the ratio of averaged fermion number density over electron number density in the medium. In the sun $Y_{u} \approx 2$ and $Y_{d} \approx 1$ and in the earth $Y_{u} \approx Y_{d} \approx 3$.

In order to study the effect of the NSI on the solar neutrinos, we can reduce the $3 \times 3$ Hamiltonian in Eq. (3), to an effective $2 \times 2$ Hamiltonian to obtain the survival probability. To study the propagation of solar neutrinos it is possible to work in the one mass dominance approximation that is given by [39] $\frac{\Delta m_{31}^{2}}{E_{\nu}} \gg G_{F} N_{e}$. In this approximation, the effective $2 \times$ 2 Hamiltonian is given by [40] 


$$
\begin{aligned}
H_{\text {vac }}^{\text {eff }}= & \frac{\Delta m_{21}^{2}}{4 E_{\nu}}\left(\begin{array}{cc}
-\cos 2 \theta_{12} & \sin 2 \theta_{12} \\
\sin 2 \theta_{12} & \cos 2 \theta_{12}
\end{array}\right), \\
H_{\text {mat }}^{\text {eff }}= & \sqrt{2} G_{F} N_{e}(r)\left(\begin{array}{cc}
c_{13}^{2} & 0 \\
0 & 0
\end{array}\right) \\
& +\sqrt{2} G_{F} \sum_{f} N_{f}(r)\left(\begin{array}{cc}
-\epsilon_{D}^{f} & \epsilon_{N}^{f} \\
\epsilon_{N}^{f *} & \epsilon_{D}^{f}
\end{array}\right) .
\end{aligned}
$$

The coefficients $\epsilon_{D}^{f}$ and $\epsilon_{N}^{f}$ are given concerning the original parameters $\epsilon_{\alpha \beta}^{f}$ as the following [15]

$$
\begin{aligned}
\epsilon_{D}^{f}= & -\frac{c_{13}^{2}}{2}\left(\epsilon_{e e}^{f}-\epsilon_{\mu \mu}^{f}\right)+\frac{s_{23}^{2}-s_{13}^{2} c_{23}^{2}}{2}\left(\epsilon_{\tau \tau}^{f}-\epsilon_{\mu \mu}^{f}\right) \\
& +\operatorname{Re}\left[c_{13} s_{13} e^{i \delta}\left(s_{23} \epsilon_{e \mu}^{f}+c_{23} \epsilon_{e \tau}^{f}\right)-\left(1+s_{13}^{2}\right) c_{23} s_{23} \epsilon_{\mu \tau}^{f}\right],
\end{aligned}
$$

$$
\begin{aligned}
\epsilon_{N}^{f}= & c_{13}\left(c_{23} \epsilon_{e \mu}^{f}-s_{23} \epsilon_{e \tau}^{f}\right) \\
& +s_{13} e^{-i \delta}\left[s_{23}^{2} \epsilon_{\mu \tau}^{f}-c_{23}^{2} \epsilon_{\mu \tau}^{f *}+c_{23} s_{23}\left(\epsilon_{\tau \tau}^{f}-\epsilon_{\mu \mu}^{f}\right)\right] .
\end{aligned}
$$

Then the effective Hamiltonian can be diagonalized as [41]

$$
U^{\prime}=\left(\begin{array}{cc}
\cos \tilde{\theta}_{12} & \sin \tilde{\theta}_{12} e^{-i \phi} \\
-\sin \tilde{\theta}_{12} e^{i \phi} & \cos \tilde{\theta}_{12}
\end{array}\right),
$$

where

$$
\tan 2 \tilde{\theta}_{12}=\frac{\left|\sin 2 \theta_{12}+2 \hat{A}_{E} \epsilon_{N}\right|}{\cos 2 \theta_{12}-\hat{A}_{E}\left(c_{13}^{2}-2 \epsilon_{D}\right)},
$$

and

$$
\phi=-\operatorname{Arg}\left(\sin 2 \theta_{12}+2 \hat{A}_{E} \epsilon_{N}\right) .
$$

Considering this effective Hamiltonian and one mass dominance approximation, survival probability reduces to [40]

$$
P(E)=\frac{1}{2} c_{13}^{4}\left[1+\cos 2 \theta_{12} \cos 2 \bar{\theta}_{12}^{m}(E)\right]+s_{13}^{4},
$$

where $\cos 2 \bar{\theta}_{12}^{m}$ is the mixing parameter averaged over the boron neutrino production region in the Sun and is given by [42]:

$$
\cos 2 \bar{\theta}_{12}^{m} \approx \frac{\cos 2 \theta_{12}-c_{13}^{2} \bar{\epsilon}_{\odot}}{\sqrt{\left(\cos 2 \theta_{12}-c_{13}^{2} \bar{\epsilon}_{\odot}\right)^{2}+\sin ^{2} 2 \theta_{12}}} .
$$

Here

$$
\bar{\epsilon}_{\odot} \equiv \frac{2 \bar{V}_{\odot} E}{\Delta m_{21}^{2}}
$$

and $\bar{V}_{\odot}$ is the averaged matter potential in the ${ }^{8} \mathrm{~B}$ neutrino production region.

\section{B. Day-night asymmetry}

In principle, matter effects on neutrino oscillation can induce a difference between day and night rates. This occurs because neutrinos which are detected during the day, travel only through the Sun, while neutrinos detected during the night pass through the Earth's matter in addition to propagating through the Sun. This difference is quantified by the so-called day-night asymmetry. The difference of survival probability during night and day is given by $[41,43]$

$$
\begin{aligned}
\Delta P(E, \eta) & =P_{N}-P_{D} \\
& =\kappa(E)\left[\int_{0}^{L} d x V(x) \sin \phi^{m}(L-x, E)+I_{2}\right] .
\end{aligned}
$$

Here

$$
\begin{aligned}
\kappa(E) \equiv & -\frac{1}{2} c_{13}^{4} \cos 2 \tilde{\theta}_{12}^{s} \sin 2 \theta_{12}\left(\sin 2 \theta_{12}\left(c_{13}^{2}-2 \epsilon_{D}^{E}\right)\right. \\
& \left.+2 \cos 2 \theta_{12} \epsilon_{N}^{E}\right)
\end{aligned}
$$

and the second term $I_{2}$ is a correction of the order $\epsilon^{2}$ and is given by

$$
I_{2}=\frac{1}{2} \cos 2 \theta_{12}\left(\int_{0}^{L} d x V(x) \cos \phi^{m}(L-x)\right)^{2},
$$

$\phi^{m}(L-x, E)$ is the adiabatic phase acquired from a given point of trajectory $x$ to a detector at $\mathrm{L}$ and is given by [43]

$$
\phi^{m}(L-x, E) \equiv \int_{x}^{L} d x \Delta_{21}^{m}(x)
$$

where

$$
\Delta_{21}^{m}=\Delta_{21} \sqrt{\left(\cos 2 \theta_{21}-\left(c_{13}^{2}-2 \epsilon_{D}^{E}\right) a_{C C}^{E}\right)^{2}+\left|\sin 2 \theta_{12}+2 a_{C C}^{E} \epsilon_{N}^{E}\right|^{2}} \approx \Delta_{21}\left(1-c_{13}^{2} \cos 2 \theta_{12} a_{C C}^{E}\right)
$$

and $a_{C C}^{E} \equiv 2 V(x) E / \Delta m_{21}^{2}$ and $\Delta_{21} \equiv \Delta m_{21}^{2} / 4 E$. As discussed in [43] $I_{2}$ is very small and we can neglect it in our calculations. For a constant density 


$$
\Delta P(E, \eta)=-\frac{1}{2} c_{13}^{6} \cos 2 \bar{\theta}_{12}^{\odot}(E) \sin ^{2} 2 \theta_{12} \times\left(\frac { a _ { C C } } { 1 - c _ { 1 3 } \operatorname { c o s } ( 2 \theta _ { 1 2 } ) a _ { C C } } \left(1-\cos \left(L \Delta_{21}\left(1-c_{13} \cos \left(2 \theta_{12}\right) a_{C C}\right)\right)\right.\right.
$$

where $\eta$ is the nadir angle and

$$
L=\cos \eta
$$

Considering the effective resolution function $g\left(E_{r}, E\right)^{\prime}=$ $g\left(E_{r}, E\right) \sigma(E) f_{B}(E)$, where $\sigma(E)$ is the neutrino interaction cross section and $f_{B}(E)$ is the flux, and plugging expression for $\Delta P(E)$, we have the following integral

$$
\begin{aligned}
I_{\Delta}\left(E_{r}\right) & \equiv \int d E g_{\nu}^{\prime}\left(E_{r}, E\right) \Delta P(E) \\
& =\int_{0}^{L} d x V(x) \int_{0}^{E_{B}} d E g_{\nu}^{\prime}\left(E_{r}, E\right) \sin \phi^{m}(L-x, E)
\end{aligned}
$$

where we permuted integration over $x$ and $E$. Let us introduce the attenuation factor $F(L-x)$ substituting the integral over $E$ in Eq. (24) by

$$
\begin{aligned}
F( & (-x) \sin \phi^{m}\left(L-x, E_{r}\right) \\
& =\int d E g_{\nu}^{\prime}\left(E_{r}, E\right) \sin \phi^{m}(L-x, E) .
\end{aligned}
$$

In general, this equality cannot be satisfied, but it is valid for special cases and under integral over $x$. Then the integral (24) becomes

$$
I_{\Delta}\left(E_{r}\right)=\int d x V(x) F(L-x) \sin \phi^{m}\left(L-x, E_{r}\right)
$$

For the ideal resolution, $g\left(E_{r}, E\right)=\delta\left(E_{r}-E\right)$, the Eq. (25) gives $F(L-x)=1$ which means that attenuation is absent.

For the Gaussian energy resolution function the attenuation factor is given by

$$
F(d) \simeq e^{-2\left(\frac{d}{\lambda_{\text {att }}}\right)^{2}}
$$

where

$$
\lambda_{\text {att }} \equiv l_{\nu} \frac{E}{\pi \sigma_{E}}
$$

is the attenuation length, and $l_{\nu}$ is the oscillation length in vacuum

$$
l_{\nu}=\frac{4 \pi E}{\Delta m_{21}^{2}}
$$

As can be seen in Eq. (27), for $d$ much larger than $\lambda_{\text {att }}$ (remote deep interiors), $F(d)$ goes to zero, while for $d$ and $\lambda_{\text {att }}$ at same order (the shallower interior), $F(d)$ becomes large; Thus, day-night asymmetry depends on the shallower interior more than deeper interior of the Earth.

Day-night asymmetry is defined as

$$
A_{N D}\left(E_{r}, \eta\right) \equiv \frac{N_{N}}{N_{D}}-1
$$

where

$$
N_{D(N)}=A \int d E g_{\nu}\left(E^{r}, E\right) \sigma(E) f_{B}(E) \Delta P(E)_{D(N)}
$$

where $\mathrm{A}$ is the factor which includes characteristics of detection.

The averaged over the year asymmetry is given by integrating $A_{D N}$ multiplied by the exposure (weight) function $W(\eta)$ over nadir angle.

$$
\bar{A}_{D N}=\int d \eta W(\eta) A_{D N}(\eta)
$$

\section{NSI detection}

The differential cross section of neutrino electron scattering as a function of electron kinetic energy is well known and is given by [20]

$$
\begin{aligned}
& \frac{d \sigma}{d T}\left(E_{\nu}, T_{e}\right) \\
& \quad=\frac{2 G_{F}^{2} m_{e}}{\pi}\left[\left(g_{1}\right)^{2}+\left(g_{2}\right)^{2}\left(1-\frac{T_{e}}{E_{\nu}}\right)^{2}-g_{1} g_{2} \frac{m_{e} T_{e}}{E_{\nu}^{2}}\right],
\end{aligned}
$$

where $T_{e}$ is the electron recoil energy, $m_{e}$ is the electron mass, and $E_{\nu}$ is the initial neutrino energy. and $g_{1}, g_{2}$ are related to the SM neutral current couplings of the electron and are given by

$$
\begin{gathered}
g_{1}^{\nu_{e}}=g_{2}^{\bar{\nu}_{e}}=\frac{1}{2}+\sin ^{2} \theta_{W}=0.73 \\
g_{2}^{\nu_{e}}=g_{1}^{\bar{\nu}_{e}}=g_{2}^{\nu_{\mu}}=g_{1}^{\bar{\nu}_{\mu}}=\sin ^{2} \theta_{W}=0.23 \\
g_{1}^{\nu_{\mu}}=g_{2}^{\bar{\nu}_{\mu}}=-\frac{1}{2}+\sin ^{2} \theta_{W}=-0.27 .
\end{gathered}
$$

The total cross section of neutrino electron scattering as a function of energy threshold and neutrino energy is given by 


$$
\begin{array}{r}
\sigma\left(E_{\nu}, T_{e}^{\max }\right)=\frac{2 G_{F}^{2} m_{e}}{\pi}\left[\left(g_{1}^{2}+g_{2}^{2}\right)\left(T_{e}^{\max }-T_{e}^{\mathrm{th}}\right)\right. \\
-\left(g_{2}^{2}+g_{1} g_{2} \frac{m_{e}}{2 E_{\nu}}\right) \\
\left.\left(\frac{T_{e}^{\mathrm{max}^{2}}-T_{e}^{\mathrm{t}^{2}}}{E_{\nu}}\right)+\frac{1}{3} g_{2}^{2}\left(\frac{T_{e}^{\max ^{3}}-T_{e}^{\mathrm{th}^{3}}}{E_{\nu}^{2}}\right)\right]
\end{array}
$$

where

$$
T_{e}^{\max }\left(E_{\nu}\right)=\frac{2 E_{\nu}^{2}}{m_{e}+2 E_{\nu}} .
$$

Considering the neutral current NSI with electron $g_{1}^{e}$ and $g_{2}^{e}$ modifies as following [20],

$$
\begin{aligned}
& g_{1}^{e \mathrm{NSI}}=g_{1}^{e}+\epsilon_{e e}^{e L} \\
& g_{2}^{e \mathrm{NSI}}=g_{2}^{e}+\epsilon_{e e}^{e R}
\end{aligned}
$$

\section{DETAILS OF EXPERIMENT AND OUR ANALYSIS}

In this section we discuss the main results of our analysis and discuss the possibility to constrain NSI parameters, considering the future solar neutrino experiments, namely, DUNE, HK, and MICA. For the statistical inferences we have considered ten years of data taking for each detector. Hyper-Kamiokande (HK) will detect the solar neutrinos by neutrino-electron elastic scattering with $6.5 \mathrm{MeV}$ threshold [44]. Considering 0.5 Mton fiduciary volume, we have calculated 200 events per day [45]. We have assumed the energy resolution of $\mathrm{HK}$ is $\sigma_{E} / E=0.15$. DUNE will have fiducial volume $40 \mathrm{kt}$ liquid argon. On the other hand, DUNE can detect solar neutrinos via charged current interaction

$$
\nu_{e}+{ }^{40} \mathrm{Ar} \rightarrow{ }^{40} \mathrm{~K}+e^{-} .
$$

We consider the generic form of cross sections for interaction with nuclei

$$
\sigma_{C C}(E)=A p_{e} E_{e},
$$

where $A$ is a normalization factor (irrelevant for the relative excess), $E_{e}=E_{\nu}-\Delta M, p_{e}$ is the electron momentum and $\Delta M=5.8 \mathrm{MeV}$ is the reaction threshold [46]. Notice that only $9.7 \%$ of ${ }^{8} \mathrm{~B}$ neutrinos have energy $E_{\nu}>11 \mathrm{MeV}$ but due to strong energy dependence (43) the corresponding fraction of the detected events is 0.9. We find that about 27000 events of $\nu_{e}$ will be detected annually for $E_{\nu}>11 \mathrm{MeV}$ in a $40 \mathrm{kt}$ liquid argon detector considering neutrino interaction with argon nuclei $[43,46]$. We have assumed the energy resolution of DUNE is $\sigma_{E} / E=0.07$.

MICA is a proposed detector that will be located at Amundsen-Scott South Pole station [47], in the same place as ICECUBE. In our calculations we have taken the characteristics of MICA from Ref. [47], 10 Mton fiducial mass and $10 \mathrm{MeV}$ energy threshold for the kinetic energy of the recoil electron. With these parameters, we find that about $5 \times 10^{5}$ solar $\nu e$ - scattering events are expected per year. We have assumed the energy resolution of MICA will be $\sigma_{E} / E=0.15$.

We have considered only solar boron neutrino flux from Ref. [42]. We have assumed solar neutrino parameters true value are $\Delta m_{21}^{2}=7.5 \times 10^{-5}$ and $\theta_{12}=33.5^{\circ}$. Our analysis shows that in the presence of NSI, $\Delta m_{21}^{2}$ and $\theta_{12}$ will be determined with a precision of $1.2 \times 10^{-6} \mathrm{eV}^{2}$ and $0.07^{\circ}$ respectively with JUNO after 10 years of data taking. We have considered all the details of JUNO the same as given in Refs. $[18,19]$. Since JUNO is going to determine these two parameters with very high precision, we can fix these parameters in our analysis. We have assumed the PREM model [48] for the Earth structure to calculate the day-night asymmetry. Besides, we have assumed the same details of analysis as given in Ref. [43]. For the statistical inferences we have considered Asimov data set approximation, and the true model is the standard model or $\epsilon_{\alpha \beta}^{f}=0$. For statistical inferences for oscillation of the neutrinos in the sun, we have used the chi-squared method. We have neglected the matter effect in the earth or day-night asymmetry for constraining the parameters from the effect of NSI on the oscillation in the sun. We have assumed Gaussian distribution for statistical errors of day-night asymmetry. Moreover, in all of our calculation we have assumed $\epsilon_{\alpha \beta}^{e}=\epsilon_{\alpha \beta}^{u}=\epsilon_{\alpha \beta}^{d}$.

Comparing our results with the previous constraint on $\epsilon_{D}$ and $\epsilon_{N}$ which is presented in [49], our result shows that future solar neutrino experiment can constraint $\epsilon_{D}$ and $\epsilon_{N}$ 100 times more stringent than the previous results. More accurate measurement of $\theta_{12}$ and $\Delta m_{21}^{2}$ by JUNO and a large number of events collected by future solar neutrinos are responsible for this improvement. Moreover, our results show that the constraints on $\epsilon_{e e}^{e L}$ will be approximately five times more stringent than the current constraints on $\epsilon_{e e}^{e L}$ [20] at $3 \sigma$ C.L. while the constraints on $\epsilon_{e e}^{e R}$ are in the same order.

\section{RESULTS}

We have plotted $\cos 2 \tilde{\theta}_{12}$ for different values of energy and $\epsilon$ in Fig. 1, considering $\epsilon_{\alpha \beta}^{e}=\epsilon_{\alpha \beta}^{u}=\epsilon_{\alpha \beta}^{d}$. As it is indicated in the plot, for values of $|\epsilon|$ less than 0.01 , the $\epsilon_{D}$ is indistinguishable from $\epsilon_{N}$ in solar neutrino oscillation probability. For solar neutrinos with energies more than $10 \mathrm{MeV}$, there is a degeneracy between $\epsilon_{D}$ and $\epsilon_{N}$ for values of $\epsilon$ less than 0.03. In Fig. 2, we have plotted $\cos 2 \tilde{\theta}_{12}$ 

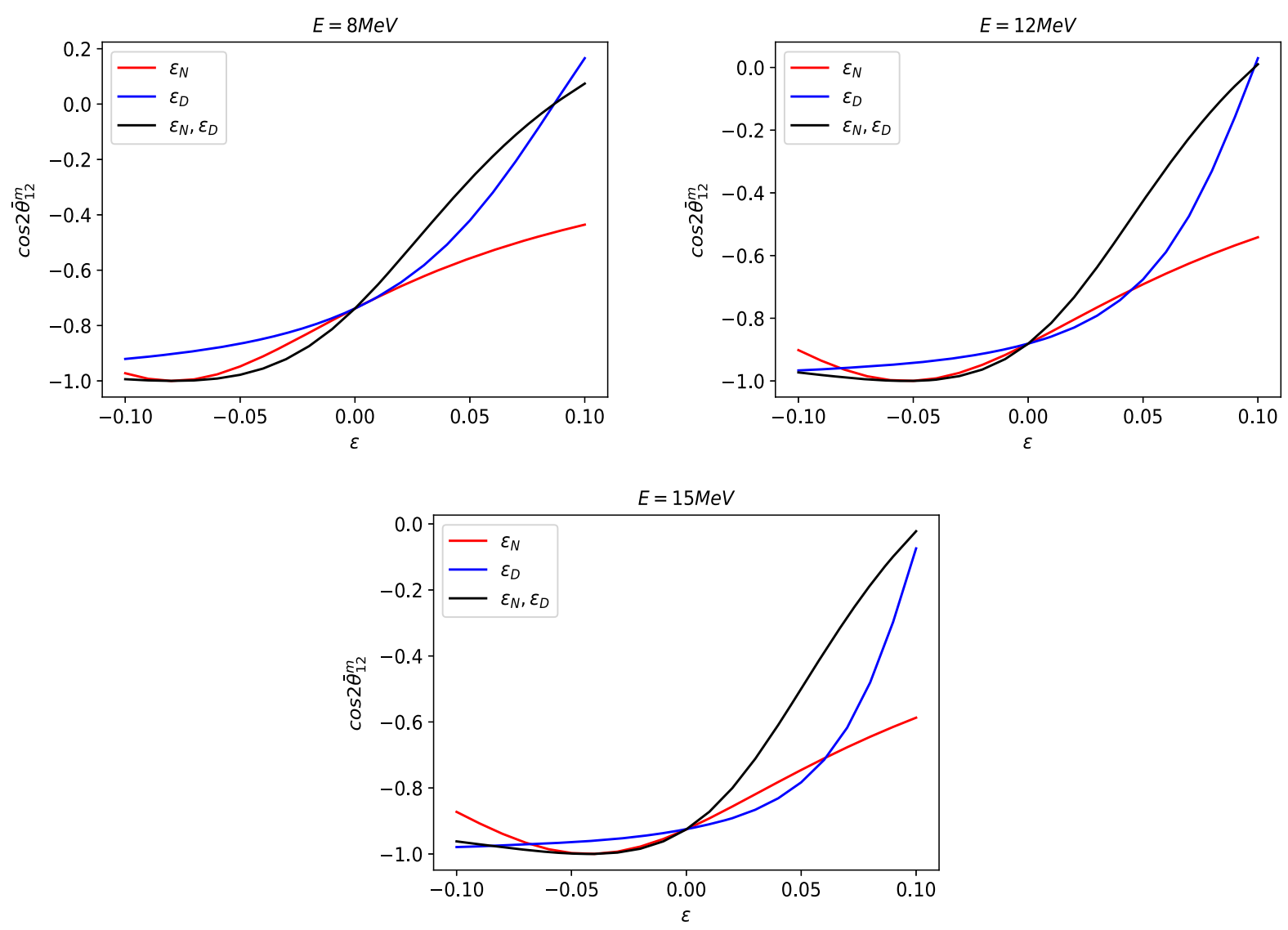

FIG. 1. $\cos 2 \tilde{\theta}_{12}$ as a function of energy $\epsilon$ is plotted for three different neutrino energies of $8,12,15 \mathrm{MeV}$. We have assumed $\epsilon_{e}=\epsilon_{u}=\epsilon_{d}$. The blue, red, and black curves are plotted assuming $\epsilon_{D} \neq 0, \epsilon_{N} \neq 0$, and $\epsilon_{D}=\epsilon_{N} \neq 0$, respectively.

versus energy considering value of $\epsilon_{D}$ and/or $\epsilon_{N}$ is equal one.

Considering different solar neutrino observatories, DUNE, HK, MICA, and combination of DUNE and HK, the constraints on $\epsilon_{D}$ and $\epsilon_{N}$ after ten years of data taking are demonstrated in Fig. 3. As it is demonstrated the constraints on $\epsilon_{D}$ and $\epsilon_{N}$ will be of order of 0.01. As

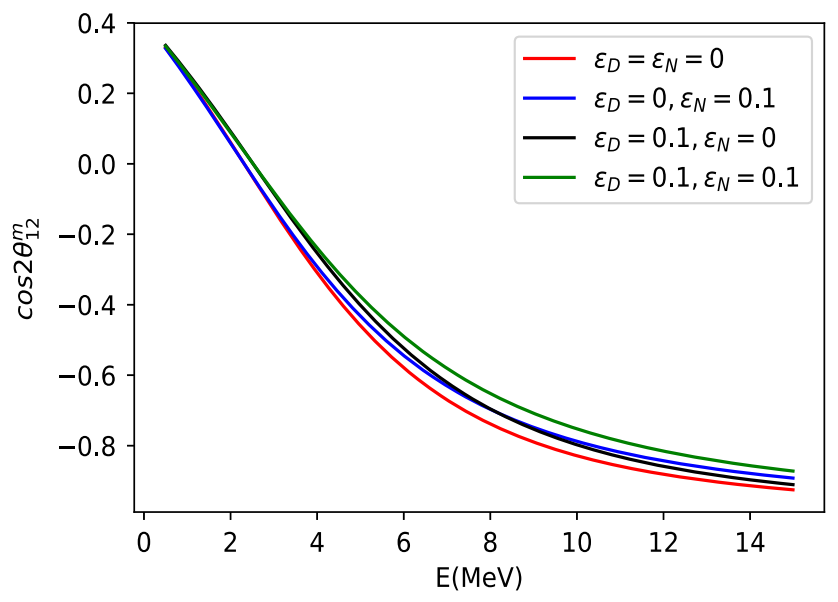

FIG. 2. $\cos 2 \tilde{\theta}_{12}$ versus energy is plotted assuming $\epsilon=0.1$. discussed before and it is demonstrated in Fig. 1, there will be a degeneracy between $\epsilon_{D}$ and $\epsilon_{N}$, and also there is a strong anticorrelation between these two parameters.

We have calculated $\Delta P(E, \eta)$ numerically, considering the PREM model, for different values of energy. Since the peak of the events approximately corresponds to $12.5 \mathrm{MeV}$, we have demonstrated the results for this value of energy and for $L=1000 \mathrm{~km}$ and $4000 \mathrm{~km}$ in Fig. 4. As it is obvious the effect of $\epsilon_{N}$ is distinguishable from the effect of $\epsilon_{D}$ and the effect of $\epsilon_{D}$ is more significant on $\Delta P(\eta)$, while in comparison to $\epsilon_{D}, \epsilon_{N}$ has a minor effect of day-night asymmetry. Considering other values of energy and baseline also leads to similar results.

We have also calculated $\bar{A}_{D N}$ for different values of $\epsilon_{D}$ and $\epsilon_{N}$ for three different experiments, DUNE, Hyper Kamiokande, and MICA, for $\theta_{12}=33.5^{\circ}$ and $\Delta m_{21}^{2}=7.5 \times 10^{-5} \mathrm{eV}^{2}$. The statistical precision measurement of DUNE, HK, and MICA will be 0.002, 0.001, and 0.0005 at $1 \sigma$ C.L. after 10 years of data taking of solar neutrinos. The uncertainty of the value of $A_{N D}$ from uncertainty of $\Delta m_{21}^{2}$ will be $0.0007,0.0004$, and 0.004 for DUNE, HK, and MICA respectively. Uncertainty of $\theta_{12}$ does not have any significant effect on $A_{N D}$. As it is indicated in Fig 5, the constraints on $\left|\epsilon_{N}\right|$, considering both 

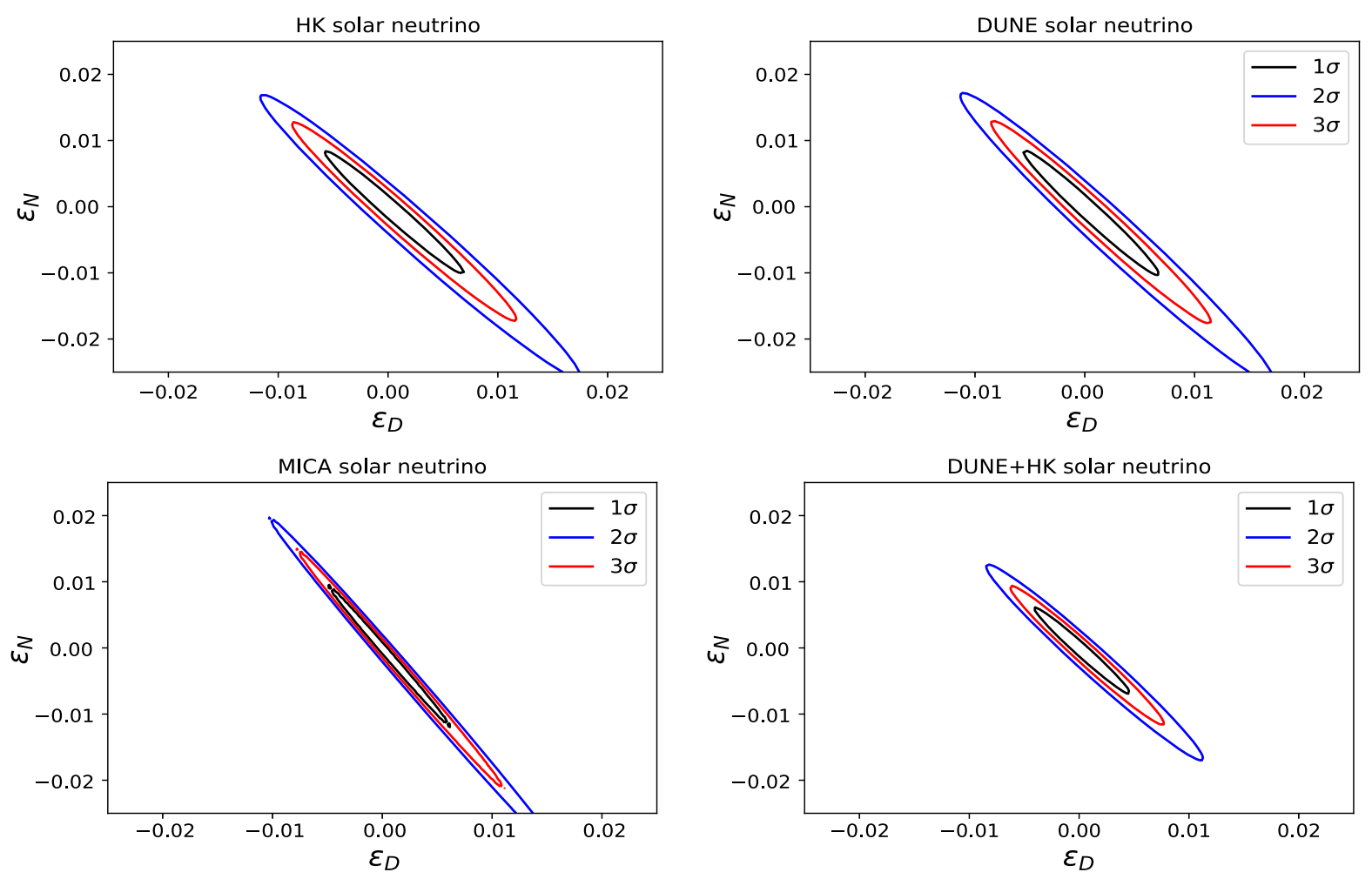

FIG. 3. $1 \sigma, 2 \sigma$, and $3 \sigma$ allowed regions for $\epsilon_{D}$ as a function of $\epsilon_{N}$ at DUNE, HK, MICA, and combination of DUNE and HK assuming $\Delta m_{21}^{2}=7.5 \times 10^{-5}$ and $\theta_{12}=33.5^{\circ}$.

the statistical precision measurement and systematic uncertainty due to measurement of $\Delta m_{21}^{2}$, will be $0.014,0.014$, and 0.007 , and the constraints on $\left|\epsilon_{D}\right|$ are $0.004,0.004$, and 0.002 , respectively with DUNE, HK and MICA.

Up to this point, in all our previous calculations for examining day-night asymmetry and oscillation of the neutrino in the sun, we have used the standard model cross section. In Fig. 6, total neutrino electron scattering cross section versus the neutrino energy is demonstrated considering different cases; The red curve is plotted

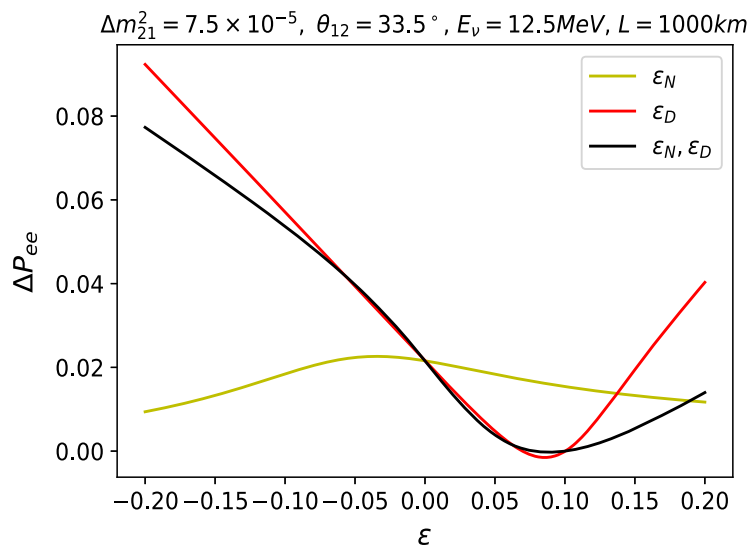

for HK experiment, considering standard model cross section for electron neutrinos, the blue curve indicates $\mathrm{NC}$ cross section for muon neutrinos, the green line is plotted considering NSI for electron neutrino interactions considering large value of $g_{R}^{e}=0.5$ and the black line is plotted considering NSI for electron neutrino interactions considering large value of $g_{L}^{e}=0.1$. We have considered the energy threshold of $6.5 \mathrm{MeV}$ for the HK experiment. In the right panel, the results are indicated for MICA experiment, considering the energy threshold of

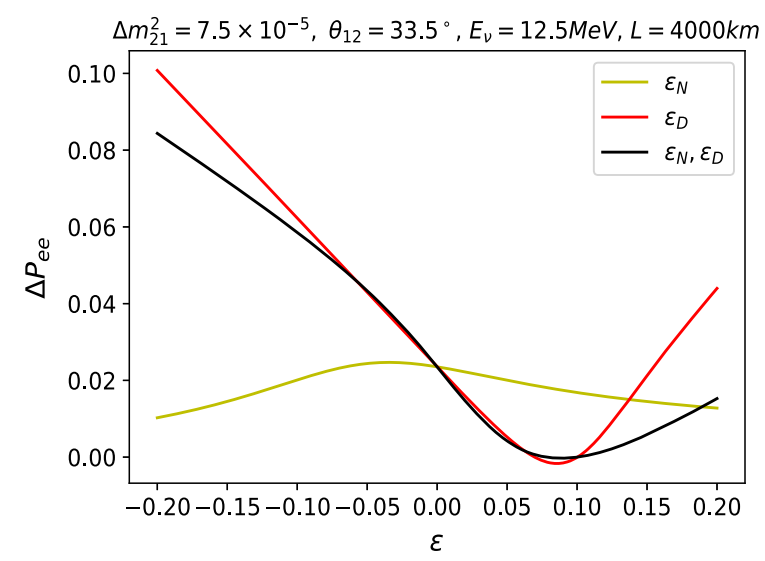

FIG. 4. The differences in survival probability during night and day is plotted versus $\epsilon$ assuming neutrinos with the energy of $12.5 \mathrm{MeV}$. We have assumed $L=1000 \mathrm{~km}$ and $L=4000 \mathrm{~km}$ in the left and in the right panel, respectively. As can be seen, $\epsilon_{D}$ has a more significant effect on $\Delta P$. 

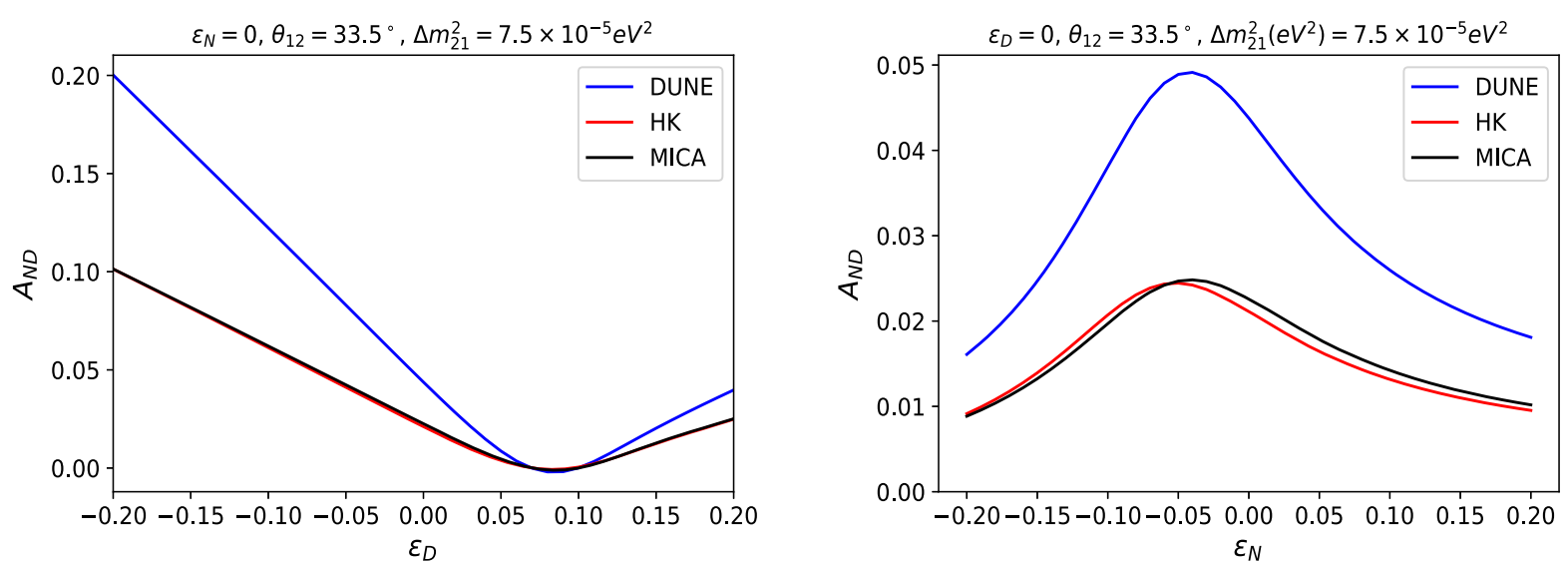

FIG. 5. $A_{N D}$ as a function of $\epsilon$ is plotted for DUNE, HK, and MICA experiments.

$10 \mathrm{MeV}$. As can be seen from the plot, the effect of $g_{R}^{e}$ is minor.

If the future experiments establish a larger day-night asymmetry, similar to the value found by current SuperKamiokande $\left(A_{N D}=3.3 \pm 1 \pm 0.5\right.$ percent) [50], considering that the prediction of best point fit of solar parameters from Kamland is 1.7 percent, such a significant difference cannot be explained by the allowed values of $\epsilon_{N}$, but it can be explained by the allowed values of $\epsilon_{D}$. Notice that both $\epsilon_{N}$ and $\epsilon_{D}$ will be constrained stringently by the oscillation of neutrinos in the Sun.

Moreover, it is interesting to investigate the effect of NSI at the detector in the simulated data for these experiments. We have demonstrated the potential of HK and MICA to constrain $\epsilon_{e e}^{e L}$ versus $\epsilon_{e e}^{e R}$ in Fig 7. As it can be seen from the plot, the constraint on $\epsilon_{e e}^{e L}$ is very stringent while in the case of $\epsilon_{e e}^{e R}$ is not.

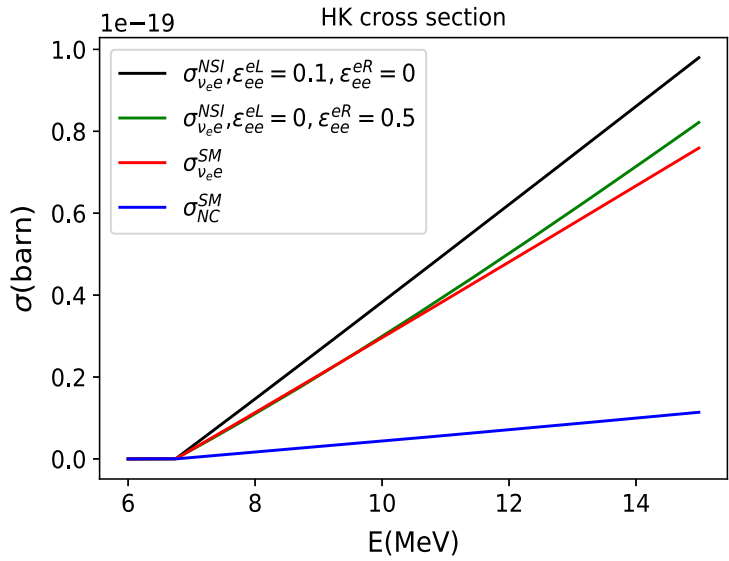

The uncertainty of $\epsilon_{e e}^{e L}$ and $\epsilon_{e e}^{e R}$ can affect HK and MICA measurement of $\epsilon_{D}$ and $\epsilon_{N}$ from oscillation of the neutrino in the Sun. In the case of DUNE near detector, since the neutrino detection is via charged current interaction, adding the NC interaction does not affect the cross-section. However, if we consider charged current NSI, the NSI parameters will be constrained stringently by DUNE near detector down to the order 0.001 as studied in ref. [13]; Thus, considering CC NSI does not affect the cross section of DUNE and in consequence measurement of $\epsilon_{e e}^{e R}$ and $\epsilon_{e e}^{e L}$.

In the case of MICA and HK, the uncertainties of the cross section and the flux do not affect day-night asymmetry. It can be seen from Eq. (30) where the uncertainties enter in both the denominator and in the numerator; Thus, the uncertainties of $\epsilon_{e e}^{e L}$ and $\epsilon_{e e}^{e R}$ do not affect the measurement of $\epsilon_{N}$ and $\epsilon_{D}$ in the day-night asymmetry. This is because cross section sensitivity to the energy is

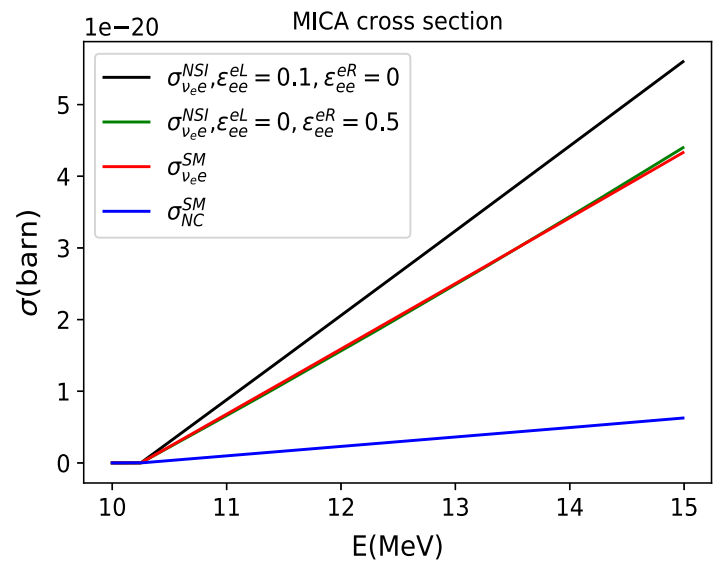

FIG. 6. Total neutrino electron scattering cross section versus the neutrino energy. In the left panel, neutrino electron scattering cross section is plotted for HK experiment with the energy threshold of $6.5 \mathrm{MeV}$. the red line is plotted considering standard model cross section for electron neutrinos, the blue line indicates NC cross section for muon neutrinos, the green line is plotted considering NSI for electron neutrino interactions considering large value of $g_{R}^{e}=0.5$ and the black line is plotted considering NSI for electron neutrino interactions considering large value of $g_{L}^{e}=0.1$. In the right panel, neutrino electron scattering cross section is plotted considering MICA experiment with the energy threshold of $10 \mathrm{MeV}$. As can be seen from the plot, the effect of $g_{R}^{e}$ is minor. 

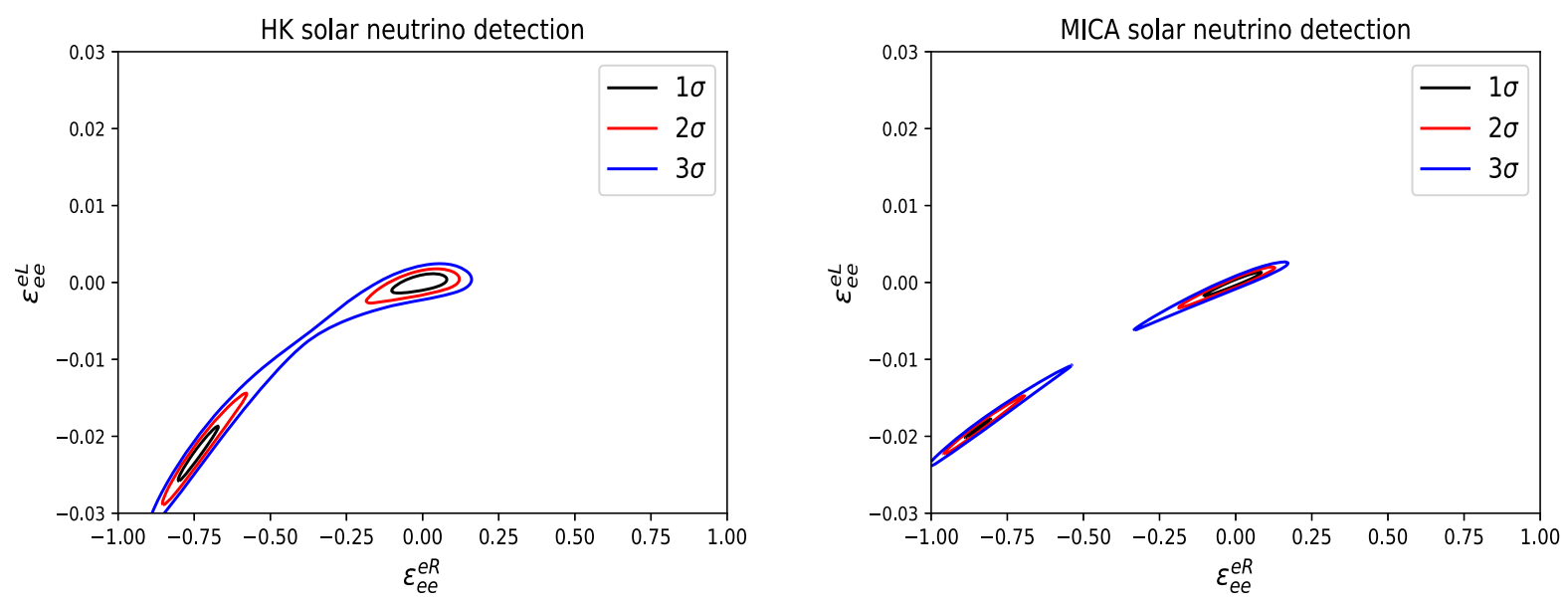

FIG. 7. $1 \sigma, 2 \sigma$ and $3 \sigma$ allowed regions for $\epsilon_{e e}^{e L}$ as a function of $\epsilon_{e e}^{e R}$ for HK (left) and MICA (right).

negligible and the normalization factors cancel out in Eq. (30); Thus, the constraints from day-night asymmetry are not affected.

Since $\epsilon_{e e}^{e L}$ has a more significant impact of neutrino electron elastic scattering cross section than $\epsilon_{e e}^{e R}$, to find the
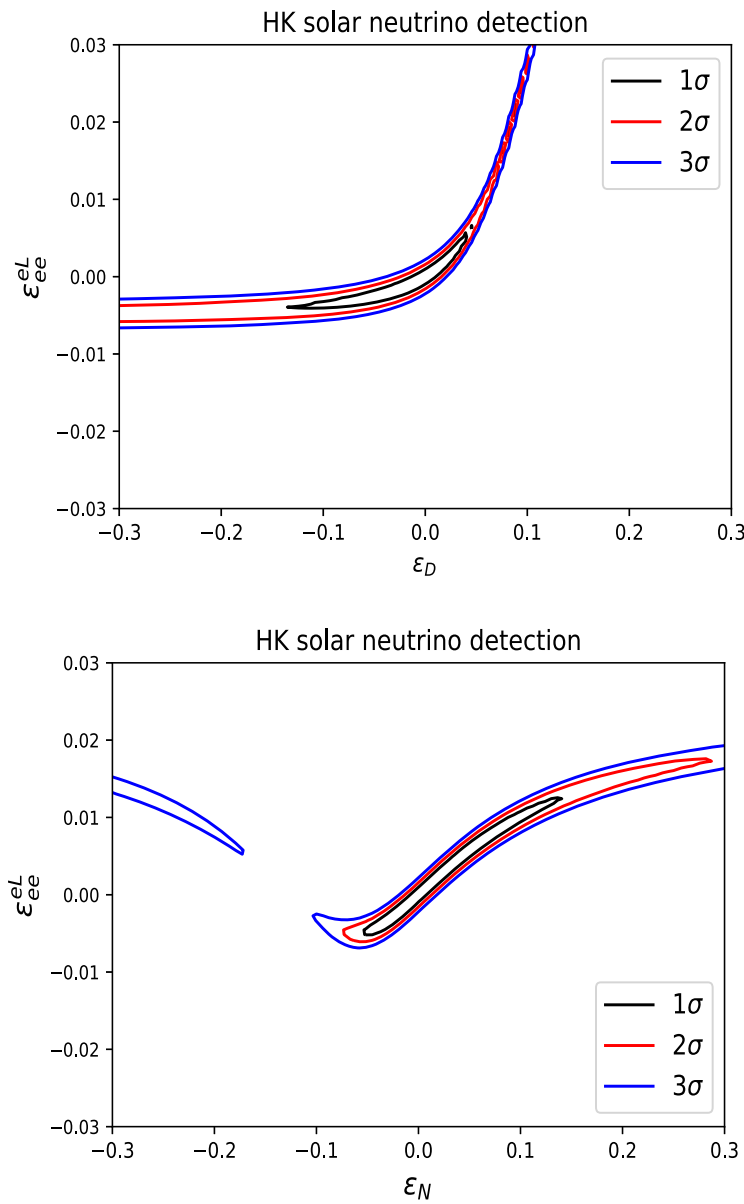

impact of cross section uncertainty on $\epsilon_{D}$ and $\epsilon_{N}$ measurements from oscillation of the neutrinos in the Sun, we can explore the potential of HK and MICA using simultaneous measurement of $\epsilon_{e e}^{e L}$ and $\epsilon_{D}$ or by simultaneous measurement of $\epsilon_{e e}^{e L}$ and $\epsilon_{N}$. The results are demonstrated in Fig. 8.
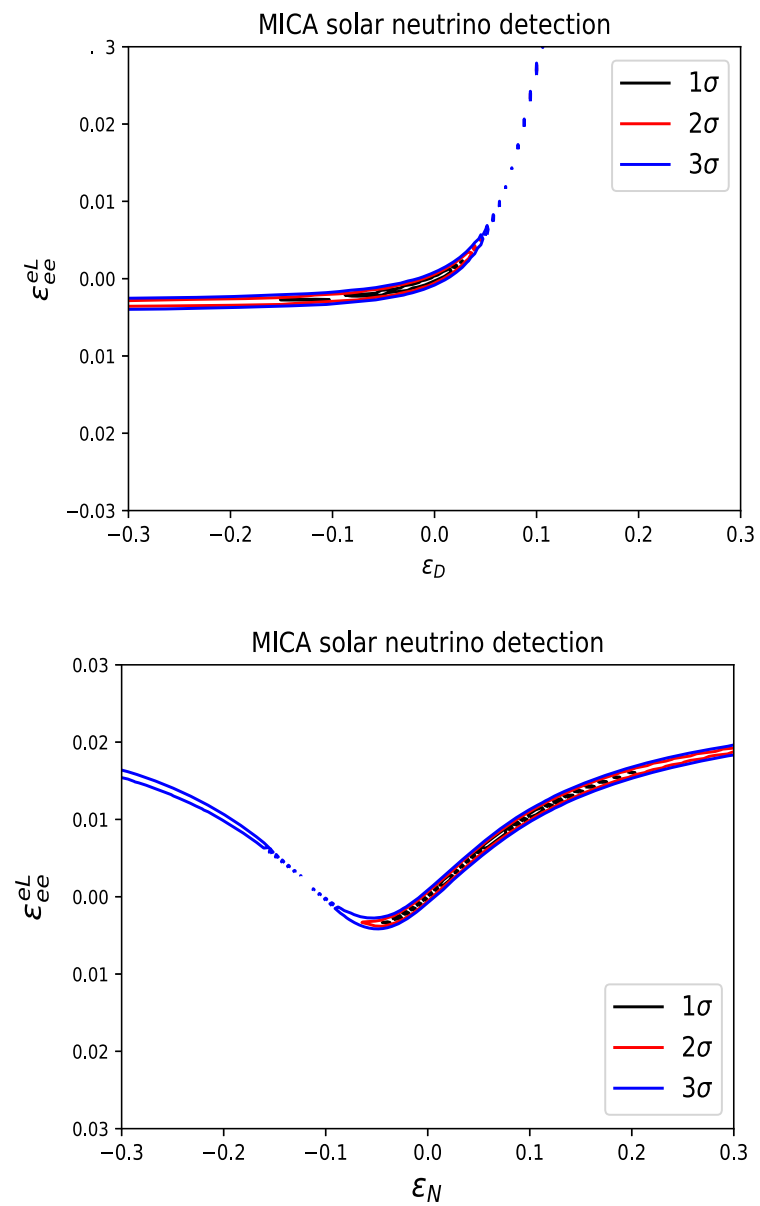

FIG. 8. $1 \sigma, 2 \sigma$ and $3 \sigma$ allowed region plotted for simultaneous measurement of $\epsilon_{D}$ and $\epsilon_{e e}^{e R}$ for HK (upper left panel) and for MICA (upper right panel). The lower panel indicates $1 \sigma, 2 \sigma$ and $3 \sigma$ allowed region for the simultaneous measurement of $\epsilon_{N}$ and $\epsilon_{e e}^{e R}$ with HK (lower left) and MICA (lower right). 
As it is shown, $\epsilon_{e e}^{e L}$ uncertainty has a huge impact on $\epsilon_{D}$ and $\epsilon_{N}$ measurement, with HK and MICA from neutrino oscillation in the Sun. However, as it is explained before, the constraints from day-night asymmetry are not affected. As it is indicated in Fig. 3, DUNE will constrain $\epsilon_{D}$ and $\epsilon_{N}$ down to 0.01 with $1 \sigma$, and $\epsilon_{N}$ and $\epsilon_{D}$ will be constrained by day-night asymmetry down to 0.01 and 0.004 , respectively; Thus, $\epsilon_{e e}^{e L}$ will be constrained down to order of 0.001 .

\section{SUMMARY}

We studied the sensitivities to NSI in the proposed nextgeneration solar neutrino observatories DUNE, HK, and MICA. The JUNO experiment will be able to measure $\Delta m_{12}^{2}$ and $\theta_{12}$ with less than one percent precision. On the other hand, having relatively low energy, the reactor experiment, JUNO is not sensitive to the standard and nonstandard matter effects. To study the effect of NSI parameters on the experimental performance, we considered this possible precise measurement of $\Delta m_{12}^{2}$ and $\theta_{12}$ by JUNO. We also assumed same NSI couplings for electron, up and down quarks $\left(\epsilon_{\alpha \beta}^{e}=\epsilon_{\alpha \beta}^{u}=\epsilon_{\alpha \beta}^{d}\right)$.

Considering neutrino oscillation in the Sun, we demonstrated the constrains on $\epsilon_{D}$ and $\epsilon_{N}$ down to order of 0.01 at $3 \sigma$ C.L. after ten years of data taking in Fig. 3 for future experiments DUNE, HK and the proposed MICA experiment in addition to the combination of DUNE and HK. We found that for the values of $|\epsilon|$ less than order of 0.01 , the $\epsilon_{D}$ is indistinguishable from $\epsilon_{N}$ in solar neutrino oscillation probability as indicated in Fig. 1; We further studied the day-night asymmetry parameters for three different experiments, DUNE, HK, and MICA. As we indicated in Fig 5, $\epsilon_{D}$ is significantly affected on day-night asymmetry while $\epsilon_{N}$ is not. We discussed that for the case of larger value of day-night asymmetry that may be established by future experiments, the allowed values of $\epsilon_{N}$ cannot explain such a huge difference while it can be explained by the allowed values of $\epsilon_{D}$.

In addition, we studied the effect of NSI at the detector for the simulated data for these experiments. We demonstrated the potential of HK and MICA to constrain NSI. Our results show that while $\epsilon_{e e}^{e R}$ is weakly constrained, the constraint on $\epsilon_{e e}^{e L}$ is very stringent. We further studied the potential of HK and MICA simultaneous measurement of $\epsilon_{e e}^{e L}$ and $\epsilon_{D}$ and simultaneous measurement of $\epsilon_{e e}^{e L}$ and $\epsilon_{N}$. We found that for HK and MICA experiments, $\epsilon_{e e}^{e L}$ uncertainty has a significant effect on $\epsilon_{D}$ and $\epsilon_{N}$ measurement considering neutrino oscillation in the Sun.

\section{ACKNOWLEDGMENTS}

The authors are thankful to the anonymous referee for her/his very useful remarks. The authors are grateful to E. Fernndez Martnez for useful discussion. This project has received funding from the European Union's Horizon 2020 research and innovation programme under the Marie Skłodowska-Curie grant agreement No. 674896 and No. 690575. We are very grateful to the IFT institute, UAM University for warm and generous hospitality during this project. P. B thanks Iran Science Elites Federation Grant No. 11131.
[1] C. Patrignani et al. (Particle Data Group), Chin. Phys. C 40, 100001 (2016).

[2] F. Capozzi, E. Di Valentino, E. Lisi, A. Marrone, A. Melchiorri, and A. Palazzo, Phys. Rev. D 95, 096014 (2017).

[3] P. F. de Salas, D. V. Forero, C. A. Ternes, M. Tortola, and J. W. F. Valle, Phys. Lett. B 782, 633 (2018).

[4] I. Esteban, M. C. Gonzalez-Garcia, M. Maltoni, I. MartinezSoler, and T. Schwetz, J. High Energy Phys. 01 (2017) 087.

[5] Z. Djurcic et al. (JUNO Collaboration), arXiv:1508.07166.

[6] S. Fukasawa, M. Ghosh, and O. Yasuda, Phys. Rev. D 95, 055005 (2017).

[7] R. Acciarri et al. (DUNE Collaboration), arXiv:1512.06148.

[8] M. M. Guzzo, A. Masiero, and S. T. Petcov, Phys. Lett. B 260, 154 (1991).

[9] P. I. Krastev and S. T. Petcov, Phys. Lett. B 299, 99 (1993).

[10] M. C. Gonzalez-Garcia, M. M. Guzzo, P. I. Krastev, H. Nunokawa, O. L. G. Peres, V. Pleitez, J. W. F. Valle, and R. Zukanovich Funchal, Phys. Rev. Lett. 82, 3202 (1999).
[11] A. Friedland and C. Lunardini, Phys. Rev. D 74, 033012 (2006).

[12] M. Masud, A. Chatterjee, and P. Mehta, J. Phys. G 43, 095005 (2016).

[13] P. Bakhti, A. N. Khan, and W. Wang, J. Phys. G 44, 125001 (2017).

[14] I. Esteban, M. C. Gonzalez-Garcia, A. Hernandez-Cabezudo, M. Maltoni, and T. Schwetz, J. High Energy Phys. 01 (2019) 106.

[15] M. C. Gonzalez-Garcia and M. Maltoni, J. High Energy Phys. 09 (2013) 152.

[16] L. Wolfenstein, Phys. Rev. D 17, 2369 (1978).

[17] Z. Djurcic et al. (JUNO Collaboration), arXiv:1508.07166.

[18] P. Bakhti and Y. Farzan, J. High Energy Phys. 07 (2014) 064.

[19] P. Bakhti and Y. Farzan, J. High Energy Phys. 10 (2013) 200.

[20] A. Bolanos, O. G. Miranda, A. Palazzo, M. A. Tortola, and J. W. F. Valle, Phys. Rev. D 79, 113012 (2009). 
[21] M. C. Gonzalez-Garcia, M. M. Guzzo, P. I. Krastev, H. Nunokawa, O. L. G. Peres, V. Pleitez, J. W. F. Valle, and R. Zukanovich Funchal, Phys. Rev. Lett. 82, 3202 (1999).

[22] P. Lipari and M. Lusignoli, Phys. Rev. D 60, 013003 (1999).

[23] N. Fornengo, M. C. Gonzalez-Garcia, and J. W. F. Valle, J. High Energy Phys. 07 (2000) 006.

[24] N. Fornengo, M. Maltoni, R. T. Bayo, and J. W. F. Valle, Phys. Rev. D 65, 013010 (2001).

[25] Z. Berezhiani and A. Rossi, Phys. Lett. B 535, 207 (2002).

[26] S. Davidson, C. Pena-Garay, N. Rius, and A. Santamaria, J. High Energy Phys. 03 (2003) 011.

[27] C. Biggio, M. Blennow, and E. Fernandez-Martinez, J. High Energy Phys. 08 (2009) 090.

[28] A. Friedland, C. Lunardini, and C. Pena-Garay, Phys. Lett. B 594, 347 (2004).

[29] A. Palazzo and J. W. F. Valle, Phys. Rev. D 80, 091301 (2009).

[30] J. Barranco, O. G. Miranda, C. A. Moura, and J. W. F. Valle, Phys. Rev. D 73, 113001 (2006).

[31] J. Barranco, O. G. Miranda, C. A. Moura, and J. W. F. Valle, Phys. Rev. D 77, 093014 (2008).

[32] F. J. Escrihuela, O. G. Miranda, M. A. Tortola, and J. W. F. Valle, Phys. Rev. D 80, 105009 (2009); 80, 129908(E) (2009).

[33] A. de Gouvea and K. J. Kelly, Nucl. Phys. B908, 318 (2016).

[34] R. Adhikari, S. Chakraborty, A. Dasgupta, and S. Roy, Phys. Rev. D 86, 073010 (2012).

[35] W. Buchmüller and D. Wyler, Nucl. Phys. B268, 621 (1986).
[36] Z. Berezhiani and A. Rossi, Phys. Lett. B 535, 207 (2002).

[37] T. Ohlsson, Rep. Prog. Phys. 76, 044201 (2013).

[38] O. G. Miranda and H. Nunokawa, New J. Phys. 17, 095002 (2015).

[39] M. Guzzo, H. Nunokawa, P. de Holanda, and O. Peres, Phys. Rev. D 64, 097301 (2001).

[40] M. C. Gonzalez-Garcia and M. Maltoni, J. High Energy Phys. 09 (2013) 152.

[41] J. Liao, D. Marfatia, and K. Whisnant, Phys. Rev. D 92 , 073004 (2015)

[42] J. N. Bahcall, E. Lisi, D. E. Alburger, L. De Braeckeleer, S. J. Freedman, and J. Napolitano, Phys. Rev. C 54, 411 (1996).

[43] P. Bakhti and A. Y. Smirnov, Phys. Rev. D 101, 123031 (2020).

[44] Hyper-Kamiokande Collaboration, Reports No. KEK-PREPRINT-2016-21, No. ICRR-REPORT-701-2016-1.

[45] J. Liao, D. Marfatia, and K. Whisnant, Phys. Lett. B 771, 247 (2017).

[46] A. Ioannisian, A. Smirnov, and D. Wyler, Phys. Rev. D 96, 036005 (2017).

[47] S. Boser, M. Kowalski, L. Schulte, N. L. Strotjohann, and M. Voge, Astropart. Phys. 62, 54 (2015).

[48] A. M. Dziewonski and D. L. Anderson, Phys. Earth Planet. Inter. 25, 297 (1981).

[49] I. Esteban, M. C. Gonzalez-Garcia, M. Maltoni, I. MartinezSoler, and J. Salvado, J. High Energy Phys. 08 (2018) 180.

[50] K. Abe et al. (Super-Kamiokande Collaboration), Phys. Rev. D 94, 052010 (2016). 